\title{
Tipos de ensayos clínicos con asignación aleatoria publicados en PubMed durante $\mathbf{4 0}$ años
}

\section{Types of randomized clinical trials published in PubMed over 40 years}

\author{
Mariana Martínez-Franco, Antonio Roberto Nirta-Pérez, Jorge Hernando Donado-Gómez.• \\ Medelín (Colombia)
}

DOI: https://doi.org/10.36104/amc.2021.1884

\section{Resumen}

Introducción: existen múltiples variantes del ensayo clínico aleatorizado; según diseño: grupos paralelos, cruzado, factorial, asignación por grupos, $\mathrm{N}$ de 1, pareado, retiro, adaptativo y pragmático; y por propósito: superioridad, no inferioridad y equivalencia. Debido a esta heterogeneidad, en 1996 se introdujo el CONSORT (Consolidated Standards of Reporting Trials) para suministrar una guía para el reporte de los ensayos clínicos aleatorizados.

Objetivo: describir la tendencia de publicación de los tipos de ensayos clínicos aleatorizados durante 40 años, en relación con la publicación del CONSORT y sus extensiones.

Métodos: se utilizó la herramienta PubMed para realizar una búsqueda de ensayos clínicos aleatorizados publicados entre 1979 y 2018 , discriminándolos según las variantes descritas.

Resultados: se encontraron 472114 artículos publicados, el 90.2\% no reportó tipo de diseño y $98.2 \%$ no reportó propósito. Entre los artículos que reportaron la variante de ensayos clínicos aleatorizados, el diseño predominante fue el cruzado (5.9\%), seguido por grupos paralelos (2.34\%); mientras que el propósito más frecuente fue el de superioridad $(0.84 \%)$. Hubo un aumento en la proporción de artículos por variante publicados después del lanzamiento del CONSORT para los diseños de: grupos paralelos; diferencia de proporciones 1.89 IC 95\% (1.1-2.7); pareado 1.07 IC 95\% (0.2-1.9); pragmático 4.73 IC 95\% (4.4-5.1); y para el propósito de no inferioridad 5.97 IC $95 \%(5.6-6.3)$.

Discusión: la mayoría de los artículos sobre ensayos clínicos aleatorizados no mencionan en su título y resumen la variante de estos. La proporción de artículos que sí lo hicieron, aumentó discretamente después de la publicación del CONSORT. (Acta Med Colomb 2021; 46. DOI: https:// doi.org/10.36104/amc.2021.1884).

Palabras clave: ensayo clínico, diseño de investigaciones epidemiológicas, publicaciones, características de estudios epidemiológicos, evaluación de resultados de intervenciones terapéuticas.

\section{Abstract}

Introduction: there are several variations of randomized clinical trials. Trials can be classified by design as parallel, cross-over, factorial, assignment by group, $n$-of-1, paired, withdrawal, adaptive and pragmatic; and by purpose as superiority, non-inferiority and equivalence. Given this heterogeneity, the Consolidated Standards of Reporting Trials (CONSORT) were introduced in 1996 to provide a guideline for reporting randomized clinical trials.

Objective: to describe the publication tendency of the various types of randomized clinical trials over 40 years, with reference to the publication of CONSORT and its extensions.

Methods: the PubMed tool was used to search for randomized clinical trials published between 1979 and 2018, classifying them according to the varieties described.

Results: a total of 472,114 published articles were found; $90.2 \%$ did not report the type of design and $98.2 \%$ did not report the purpose. Among the articles that reported the variety of randomized clinical trial, the predominant design was cross-over (5.9\%), followed by parallel groups $(2.34 \%)$; while the most common purpose was superiority (0.84\%). After the launch of CONSORT, there was an increased proportion of articles published with the following designs: parallel groups; difference
Dra. Mariana Martínez-Franco: Médica Universidad de Antioquia; Dr. Antonio Roberto Nirta-Pérez: Médico Universidad de Antioquia; Dr. Jorge Hernando Donado-Gómez: Internista; Magíster Epidemiología Hospital Pablo Tobón Uribe, Docente Ocasional Universidad de Antioquia. Medellín (Colombia). Correspondencia: Dra. Mariana MartínezFranco. Medellín (Colombia).

E-Mail: mariana.martinezf@udea.edu.co Recibido: 19/V/2020 Aceptado: 9/XII/2020 
in proportions 1.89 95\% CI (1.1-2.7); paired $1.0795 \% \mathrm{CI}(0.2-1.9)$; and pragmatic $4.7395 \% \mathrm{CI}$

(4.4-5.1); and for the purpose of non-inferiority $5.9795 \%$ CI (5.6-6.3).

Discussion: most articles on randomized clinical trials do not mention their type in the title and abstract. The proportion of articles that did, increased slightly after CONSORT was published. (Acta Med Colomb 2021; 46. DOI: https://doi.org/10.36104/amc.2021.1884).

Key words: clinical trial, epidemiological study design, publications, epidemiological study characteristics, assessment of therapeutic intervention results.

\section{Introducción}

El ensayo clínico es un estudio prospectivo que compara los efectos de una intervención frente a un control en seres humanos (1).

Su historia se remonta al siglo XVIII, donde Lind esboza las características de lo que sería el primer ensayo clínico documentado, realizado con una pequeña muestra de pacientes con escorbuto (2).

Actualmente, se considera que el ensayo clínico aleatorizado es el estudio que aporta un mayor nivel de evidencia para las intervenciones sanitarias. Debido a su creciente popularidad, han surgido múltiples variantes del diseño inicial, el cual se conoce ahora como estudio de "grupos paralelos", que consiste en la selección de una muestra de pacientes y su asignación de forma aleatoria a uno de dos o más grupos (3).

Las demás variantes, incluyen:

- Asignación por grupos: es utilizado cuando la asignación por individuos no es adecuada por la posibilidad de "contaminación" de la intervención entre los participantes. Entonces se realiza una asignación por grupos o “clusters", los cuales pueden ser zonas geográficas, instituciones, o grupos de pacientes atendidos por un médico $(4,5)$.

- Cruzado (cross over): en este caso cada participante actúa como su mismo control, de forma que recibe la intervención y el comparador durante periodos distintos, y con un tiempo de lavado entre éstos $(4,5,19)$.

- N de 1: variante utilizada cuando la evidencia disponible no es útil para un paciente en particular. Por tanto se realiza un estudio cruzado con dicho paciente, de forma que él actúa como su propio control y recibe de forma alternada y aleatoria la intervención estudiada y el comparador, en varios ciclos. Toda la población se limita a un paciente y los resultados del estudio no son extrapolables más que a éste (6).

- Factorial: permite evaluar dos o más intervenciones en un único estudio. Se asignan las intervenciones aleatoriamente de forma tal que los participantes pueden no recibir ninguna intervención, solo una de ellas o eventualmente todas $(4,5,19)$.

- Pareado: en este diseño, la unidad que es aleatorizada no es un individuo sino un órgano o sitio del cuerpo, de forma que cada participante puede recibir dos o más tratamientos en diferentes lugares del cuerpo. Otros tér- minos utilizados para describirlo son "intrapersona", "de cuerpo dividido", "de cara dividida", "de boca dividida" o "contralateral" (7).

- De retiro (withdrawal): el objetivo de este, es evaluar la respuesta a la descontinuación o disminución de la dosis de una intervención, la cual ya se ha tolerado y demostrado su beneficio $(4,5)$.

- Adaptativo: esta es una de las variantes más recientes del ensayo clínico aleatorizado, consiste en permitir la variabilidad de los participantes, el tamaño de la muestra, la intervención y los desenlaces de acuerdo con los resultados preliminares que arroje el estudio. Dicha permisividad debe estar estipulada desde el protocolo de investigación (8).

- Pragmático: son diseños en los cuales se pretende maximizar la capacidad de generalización de sus resultados, por lo cual se realizan dentro de escenarios de "cuidado usual", con un amplio rango de participantes y teniendo en cuenta desenlaces clínicamente significativos como la mortalidad y la morbilidad. Su contraparte son los ensayos explicativos, el cual es un diseño más ampliamente utilizado y que cuenta con unas condiciones mucho más controladas, con las cuales se busca probar una hipótesis de causalidad, disminuyendo la capacidad de aplicación que ésta pueda tener (9).

Además de las variantes de diseño a través del tiempo han aparecido nuevos propósitos para los ensayos clínicos aleatorizados. El propósito clásico es el de superioridad, con el cual se pretende evaluar si una intervención es más eficaz que la de referencia. Sin embargo, también puede evaluarse si la intervención es no inferior, o si ambas son iguales. Éstos corresponden a los estudios de no inferioridad $y$ de equivalencia, respectivamente $(3,19)$.

Debido a la importancia que han cobrado los ensayos clínicos aleatorizados en la actualidad y a la creciente investigación en el área metodológica, un grupo de expertos ha venido desarrollando desde 1996 una herramienta que permite mejorar la calidad del reporte y disminuir los sesgos asociados a éste: el CONSORT (Consolidated Standards of Reporting Trials). Actualmente se encuentra vigente su tercera versión, la declaración del CONSORT 2010: guías actualizadas para el reporte de ensayos clínicos aleatorizados por grupos paralelos (10). Además se encuentran publicadas las extensiones de éste, con las pautas para la publicación de los diseños de asignación por grupos desde el año 2004 
$(11,12)$, no inferioridad y equivalencia desde el 2006 (13, 14), pragmático desde el 2009 (9), N de 1 desde el año 2015 (15) e intrapersona desde el año 2017 (7).

Con el presente artículo se pretende describir la tendencia de publicación de las diferentes variantes de ensayos clínicos aleatorizados durante cuarenta años, entre 1979 y 2018 y su relación con el lanzamiento de las recomendaciones del CONSORT, específicamente con respecto a la pauta del subtipo del ensayo clínico aleatorizado en el título y resumen del artículo.

\section{Material y métodos}

Estudio observacional, para el cual se utilizó la herramienta PubMed para la búsqueda de los ensayos clínicos aleatorizados publicados entre 1979 y 2018.

Se realizó una identificación inicial del número de ensayos clínicos aleatorizados publicados en este lapso, y luego se implementó una estrategia de búsqueda para cada uno de los siguientes diseños: estudio de grupos paralelos, estudio de asignación por grupos, estudio cruzado, estudio con $\mathrm{N}$ de 1, estudio factorial, estudio pareado, estudio de retiro, estudio adaptativo y estudio pragmático.

Adicionalmente se realizó una búsqueda bibliográfica con base en el propósito trabajado por los ensayos clínicos, teniendo en cuenta las tres posibilidades existentes: estudios de superioridad, de no inferioridad y de equivalencia.

Los términos de búsqueda fueron escogidos con base en los siguientes criterios:

- En el caso de los diseños para los cuales existe en la actualidad una extensión del CONSORT, se utilizó el término de búsqueda recomendado por este.

- Para los diseños aún no contemplados por el CONSORT o para los cuales no recomiendan un término en particular para su reporte, se realizó una combinación del nombre del estudio con los términos "study", "design" y "trial".
En el material suplementario podrá encontrar las estrategias de búsqueda utilizadas (Anexo 1).

Los resultados de la búsqueda fueron reportados individualmente por dos de los autores (MMF y ARNP) y se evaluó la concordancia por medio del coeficiente kappa. Los datos se presentan como frecuencias absolutas y relativas. Las comparaciones entre los datos antes y después de la publicación del CONSORT respectivo, se hicieron mediante la prueba de diferencia de proporciones. Se consideró estadísticamente significativo como un valor $\mathrm{p}<0.05$. Se empleó el paquete estadístico Epidat versión 4.2 (22).

Este estudio no requirió de la aprobación por un comité de ética, debido a que la unidad de análisis son ensayos clínicos publicados.

\section{Resultados}

De acuerdo con la búsqueda bibliográfica realizada entre 1979 y 2018, fueron publicados un total de 472114 artículos etiquetados como "ensayo clínico aleatorizado" y $94.58 \%$ se encontraba publicado en inglés, seguido por $1.7 \%$ en chino y $1.25 \%$ en alemán, los demás idiomas como el francés, español, portugués, japonés, italiano y ruso tuvieron una representación menor a 1\%. El 90.2\% (426 071) de estos artículos no mencionaba en su título o resumen la variante de diseño utilizada. El porcentaje restante se distribuyó así: $28246(5.9 \%)$ fueron de la variante cruzado, 11064 (2.34\%) de grupos paralelos, $2056(0.4 \%)$ de asignación por grupos, $1796(0.38 \%)$ de factorial, $1548(0.32 \%)$ de pragmático, 847 $(0.17 \%)$ de pareado, $246(0.05 \%)$ de adaptativo, $141(0.02 \%)$ de retiro y $99(0.02 \%)$ con $\mathrm{N}$ de 1 (Figura 1$)$.

En la búsqueda por propósito, 98.2\% (463 999) de los artículos no lo discriminaban. En el restante, se encontraron $3985(0.84 \%)$ artículos sobre ensayos clínicos aleatorizados de superioridad, $1850(0.39 \%)$ de no inferioridad y 1716 $(0.36 \%)$ de equivalencia (Figura 2). La distribución por

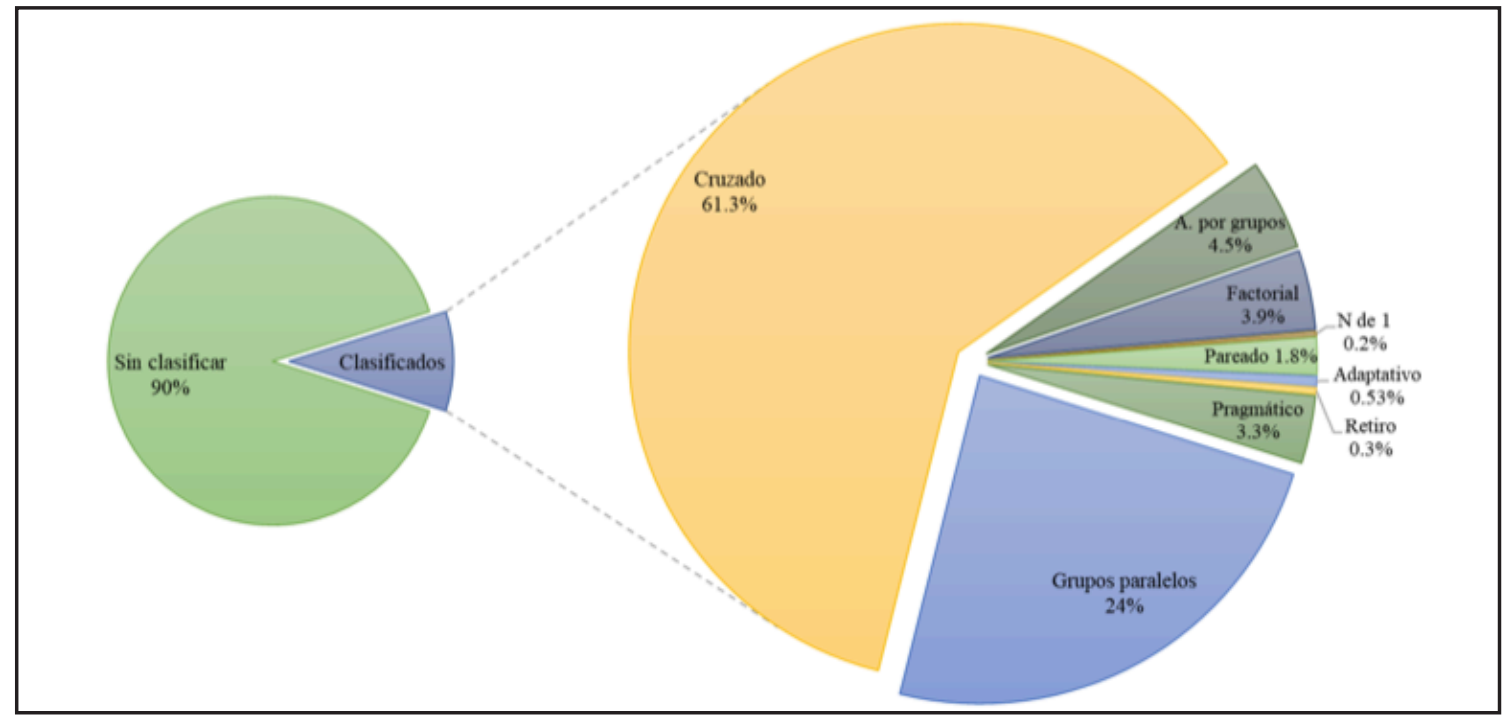

Figura 1. Distribución de los artículos sobre ensayos clínicos aleatorizados publicados entre 1979 y 2018, por diseño metodológico. 


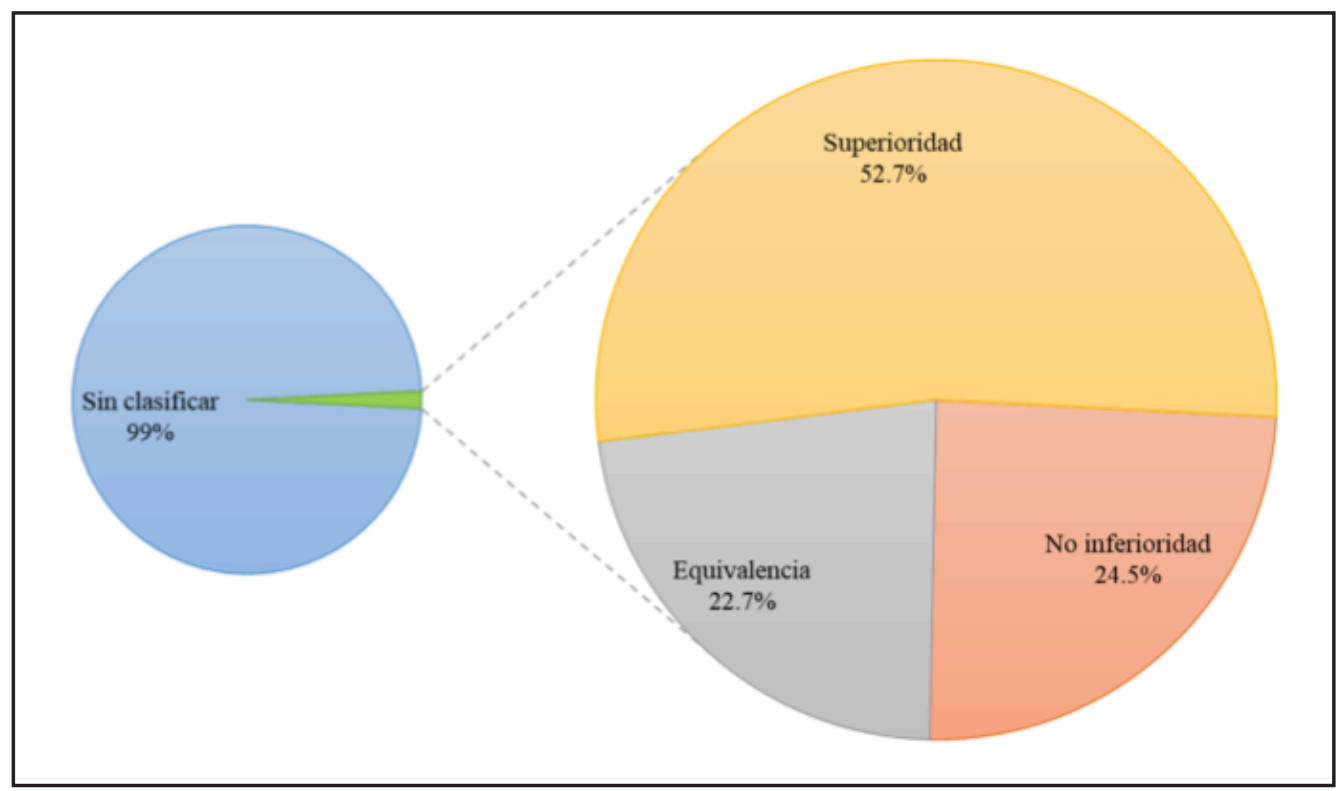

Figura 2. Distribución de los artículos sobre ensayos clínicos aleatorizados publicados entre 1979 y 2018, por propósito.

lustros de los tipos de ensayo clínico aleatorizado por diseño y propósito se pueden consultar en las Tablas 1 y 2.

En las Figuras 3 y 4 se puede observar la tendencia de publicación de las diferentes variantes de ensayos clínicos a través de los años, discriminada por lustros.

La proporción de artículos publicados que se identificaron por cada variante de ensayo clínico aleatorizado en su título o resumen, después del lanzamiento del CONSORT y sus respectivas extensiones, aumentó para los diseños de grupos paralelos, $\mathrm{N}$ de 1, pareado, pragmático y para el propósito de no inferioridad, mientras que disminuyó para el de equivalencia. Fue un cambio estadísticamente significativo en todos los casos, excepto en el de $\mathrm{N}$ de 1 y equivalencia (Tabla 3 ).

En la evaluación de la concordancia para la obtención global de los datos entre los investigadores (MMF y ARNP) se obtuvo un coeficiente kappa $(x)$ de 0.88 IC 95\% (0.82$0.95)$, lo cual implica un muy buen desempeño de acuerdo con los criterios de Cohen $(21,22)$.

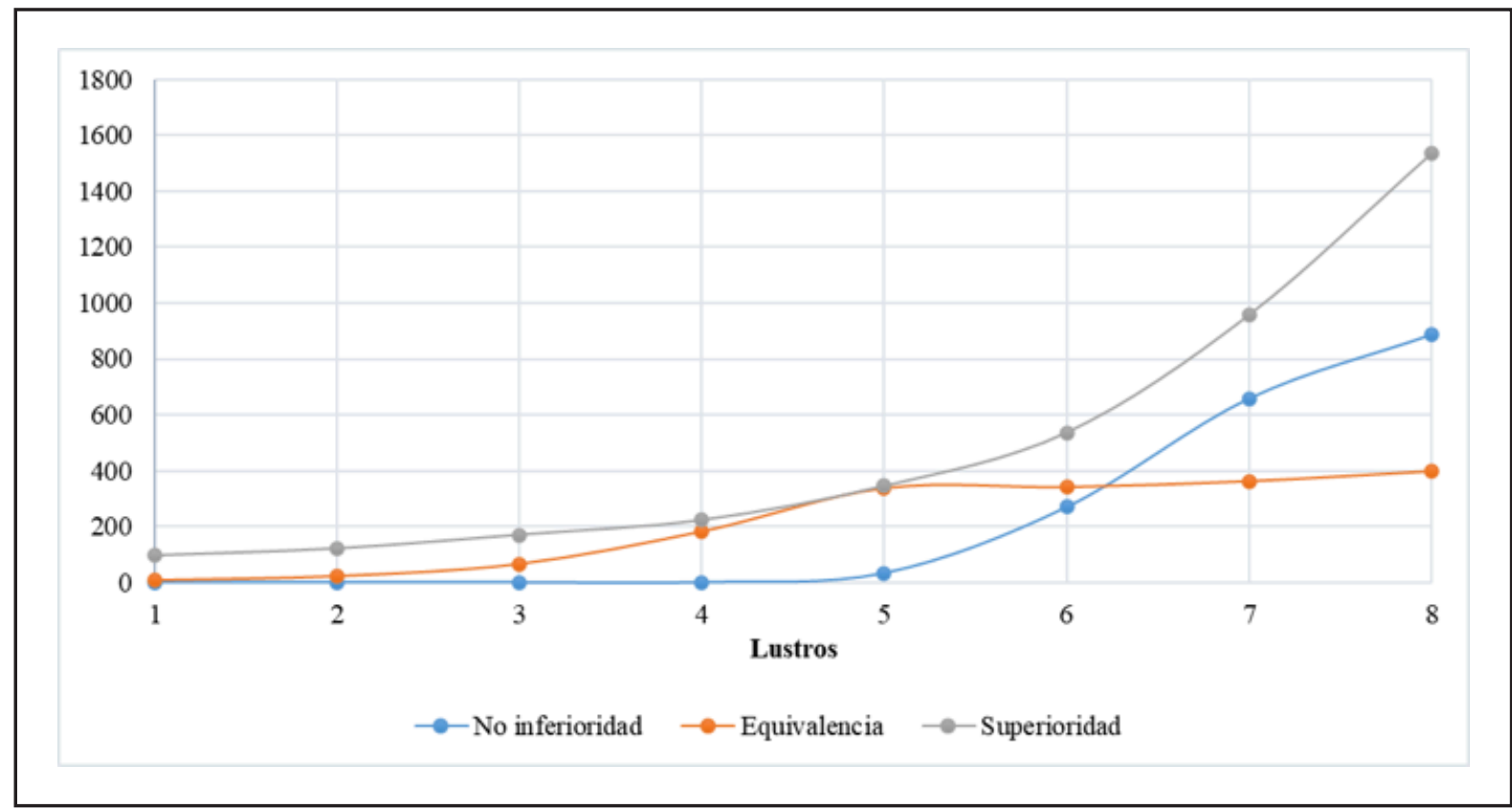

Figura 3. Tendencia por lustro de los artículos sobre ensayos clínicos aleatorizados publicados entre 1979 y 2018, clasificado según diseño metodológico. Lustro 1 (1979-1983) Lustro 2 (1984-1988), Lustro 3 (1989-1993), Lustro 4 (1994-1998), Lustro 5 (1999-2003), Lustro 6 (2004-2008), Lustro 7 (2009-2013), Lustro 8 (2014-2018). 


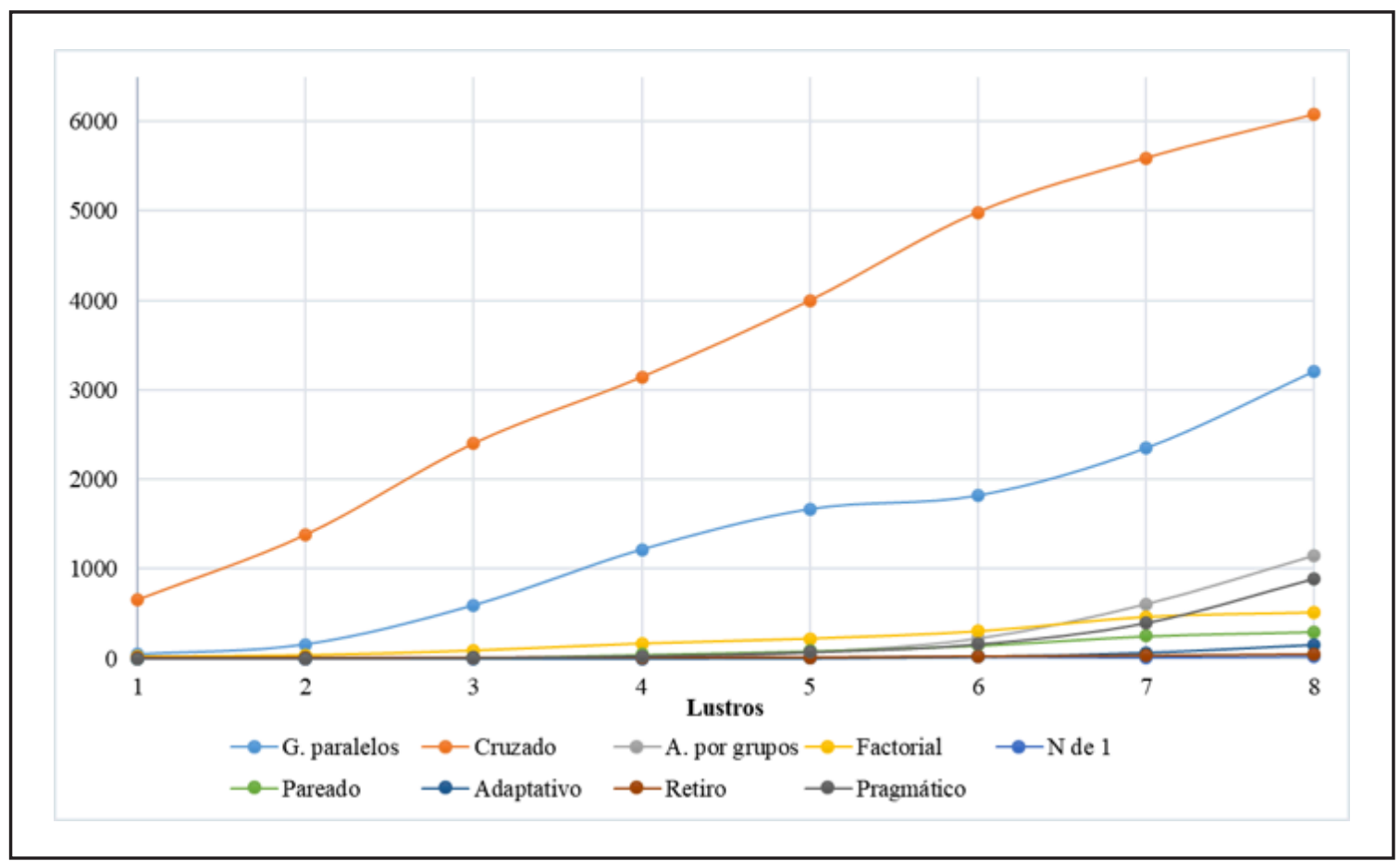

Figura 4. Tendencia por lustro de los artículos sobre ensayos clínicos aleatorizados publicados entre 1979 y 2018, clasificado según propósito. Lustro 1 (1979-1983), Lustro 2 (1984-1988), Lustro 3 (1989-1993), Lustro 4 (1994-1998), Lustro 5 (1999-2003), Lustro 6 (2004-2008), Lustro 7 (2009-2013), Lustro 8 (2014-2018).

Tabla 1. Frecuencias de tipos de ensayos clínicos aleatorizados publicados por lustro (por diseño).

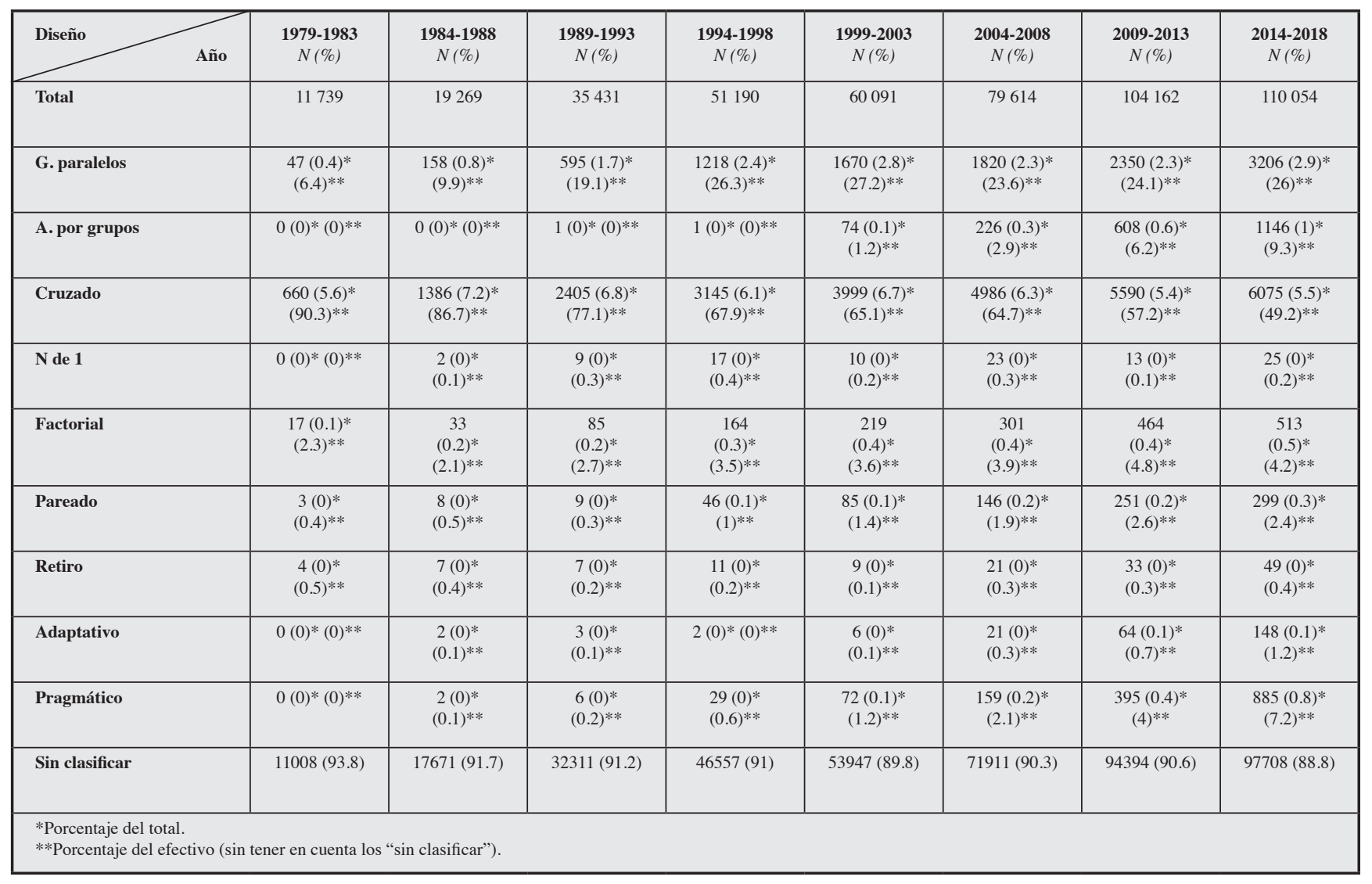


Tabla 2. Frecuencias de tipos de ensayos clínicos aleatorizados publicados por lustro (por propósito)

\begin{tabular}{|c|c|c|c|c|c|c|c|c|}
\hline Propósito & $\begin{array}{c}1979-1983 \\
N(\%)\end{array}$ & $\begin{array}{c}\text { 1984-1988 } \\
\mathrm{N}(\%)\end{array}$ & $\begin{array}{c}\text { 1989-1993 } \\
\mathrm{N}(\%)\end{array}$ & $\begin{array}{c}\text { 1994-1998 } \\
\mathrm{N}(\%)\end{array}$ & $\begin{array}{c}1999-2003 \\
\mathrm{~N}(\%)\end{array}$ & $\begin{array}{c}\text { 2004-2008 } \\
\mathrm{N}(\%)\end{array}$ & $\begin{array}{c}\text { 2009-2013 } \\
\mathrm{N}(\%)\end{array}$ & $\begin{array}{c}\text { 2014-2018 } \\
N(\%)\end{array}$ \\
\hline Total & 11739 & 19269 & 35431 & 51190 & 60091 & 79614 & 104162 & 110054 \\
\hline Superioridad & $\begin{array}{l}96(0.8)^{*} \\
(94.1)^{* *}\end{array}$ & $\begin{array}{c}121(0.6)^{*} \\
(85.2)^{* *}\end{array}$ & $\begin{array}{c}170(0.5)^{*} \\
(72.3)^{* *}\end{array}$ & $\begin{array}{c}223(0.4)^{*} \\
(55.1)^{* *}\end{array}$ & $\begin{array}{c}345(0.6)^{*} \\
(48.2)^{* *}\end{array}$ & $\begin{array}{c}536(0.7)^{*} \\
(46.7)^{* *}\end{array}$ & $\begin{array}{c}958(0.9)^{*} \\
(48.4)^{* *}\end{array}$ & $\begin{array}{c}1536(1.4)^{*} \\
(54.4)^{* *}\end{array}$ \\
\hline No inferioridad & $0(0)^{*}(0)^{* *}$ & $0(0)^{*}(0)^{* *}$ & $0(0)^{*}(0)^{* *}$ & $0(0)^{*}(0)^{* *}$ & $\begin{array}{l}33(0)^{*} \\
(4.6)^{* *}\end{array}$ & $\begin{array}{c}270(0.3)^{*} \\
(23.5)^{* *}\end{array}$ & $\begin{array}{c}659(0.6)^{*} \\
(33.3)^{* *}\end{array}$ & $\begin{array}{c}888(0.8)^{*} \\
(31.5)^{* *}\end{array}$ \\
\hline Equivalencia & $\begin{array}{c}6(0)^{*} \\
(94.1)^{* *}\end{array}$ & $\begin{array}{l}21(0.1)^{*} \\
(85.2)^{* *}\end{array}$ & $\begin{array}{l}65(0.2)^{*} \\
(72.3)^{* *}\end{array}$ & $\begin{array}{c}182(0.4)^{*} \\
(55.1)^{* *}\end{array}$ & $\begin{array}{c}338(0.6)^{*} \\
(48.2)^{* *}\end{array}$ & $\begin{array}{c}342(0.4)^{*} \\
(46.7)^{* *}\end{array}$ & $\begin{array}{c}363(0.3)^{*} \\
(48.4)^{* *}\end{array}$ & $\begin{array}{c}399(0.4)^{*} \\
(54.4)^{* *}\end{array}$ \\
\hline Sin clasificar & $11637(99.1)$ & 19127 (99.3) & $35196(99.3)$ & 50785 (99.2) & 59375 (98.8) & 78466 (98.5) & $102182(98)$ & $\begin{array}{c}107231 \\
(97.4)\end{array}$ \\
\hline
\end{tabular}

Tabla 3. Diferencia de proporciones en el patrón de publicación antes y después del CONSORT.

\begin{tabular}{|c|c|c|c|c|}
\hline \multicolumn{2}{|c|}{ Tipo de estudio } & \multirow{2}{*}{\begin{tabular}{|l} 
Proporción* (\%) \\
$4061 / 17759(22.86)$
\end{tabular}} & \multirow{3}{*}{$\begin{array}{c}\text { Diferencia de proporciones } \\
\text { (IC 95\%) }\end{array}$} & \multirow{3}{*}{$\begin{array}{l}\text { Valor P } \\
<0.001\end{array}$} \\
\hline \multirow{2}{*}{ Grupos paralelos } & Pre-CONSORT & & & \\
\hline & Pos-CONSORT & $7003 / 28284(24.75)$ & & \\
\hline \multirow{2}{*}{$\mathbf{N}$ de 1} & Pre-CONSORT & $84 / 38939(0.21)$ & \multirow{2}{*}{$0.06(-0.00-0.002)$} & \multirow{2}{*}{0.27} \\
\hline & Pos-CONSORT & $15 / 9773(0.15)$ & & \\
\hline \multirow{2}{*}{ Pareado } & Pre-CONSORT & $800 / 44410(1.80)$ & \multirow{2}{*}{$1.07(0.2-1.9)$} & \multirow{2}{*}{0.002} \\
\hline & Pos-CONSORT & $47 / 1633(2.87)$ & & \\
\hline \multirow{2}{*}{ Pragmático } & Pre-CONSORT & $323 / 25599(1.26)$ & \multirow{2}{*}{$4.73(4.4-5.1)$} & \multirow{2}{*}{$<0.001$} \\
\hline & Pos-CONSORT & $1225 / 20444(5.99)$ & & \\
\hline \multirow{2}{*}{ No inferioridad } & Pre-CONSORT & $153 / 20754(0.73)$ & \multirow{2}{*}{$5.97(5.6-6.3)$} & \multirow{2}{*}{$<0.001$} \\
\hline & Pos-CONSORT & $1697 / 25289(6.71)$ & & \\
\hline \multirow{2}{*}{ Equivalencia } & Pre-CONSORT & $802 / 20754$ (3.86) & \multirow{2}{*}{$-0.25(-0.6-0.1)$} & \multirow{2}{*}{0.16} \\
\hline & Pos-CONSORT & $914 / 25289(3.61)$ & & \\
\hline
\end{tabular}

\section{Discusión}

A través de los 40 años de publicaciones revisadas en PubMed, se encontró que más de $90 \%$ de los artículos no reportaban el tipo de ensayo clínico presentado, ni su propósito en el título o resumen (abstract). Sin embargo, se evidenció un aumento discreto, pero estadísticamente significativo en la tasa del reporte de estas características en los años posteriores al lanzamiento del CONSORT y sus extensiones. Esto indica que las recomendaciones sobre la publicación de ensayos clínicos dictadas por el CONSORT están en proceso de ser adoptadas por la comunidad científica, aunque como ha sido demostrado en estudios previos, no se ha logrado una adherencia aceptable $(16-18,20)$.
Por otra parte, sorprende el hallazgo de que, entre los artículos que reportaron su clasificación, la mayoría está representada por el estudio cruzado. Se esperaba que el diseño predominante fuera el de grupos paralelos al ser el modelo inicial, aunque el diseño cruzado es conocido que es mayormente empleado en ciencias básicas que en clínicas (3). No obstante, una de las posibilidades es que la mayoría de los artículos sin clasificar correspondan al diseño de grupos paralelos, que al ser el modelo de base para los demás, se asume como tal cuando no se específica uno alternativo.

Como se esperaba la tendencia de publicación de todos los ensayos clínicos aleatorizados fue en aumento progresivo a través de los años. Alrededor de 1990 se introdujo mayor 
variedad a éstos, con el creciente reporte de los diseños de asignación por grupos, $\mathrm{N}$ de 1, pareado, adaptativo, de retiro, pragmático y factorial. Antes de esta fecha solo las variantes cruzado y de grupos paralelos eran reportadas como tal.

Los ensayos clínicos de superioridad continúan aumentando a través del tiempo y representan la mayoría de los artículos que reportan su propósito. Los artículos sobre ensayos clínicos aleatorizados de no inferioridad hicieron su aparición alrededor del año 2000 y su reporte se encuentra también en aumento, mientras que los de equivalencia han venido con tendencia a la estabilización desde entonces.

Como debilidades del estudio, no se revisaron los textos completos de los artículos y no se consultó sino una base de datos bibliográfica, sin embargo, PubMed es la más importante a nivel global. Además, no se realizó control de duplicados, por lo cual la cifra actual de ensayos clínicos aleatorizados probablemente se encuentra sobreestimada; sin embargo, dadas las herramientas para identificar publicaciones duplicadas existentes, esto es cada vez menos frecuente, y esto no justifica la falta de adherencia al CONSORT evidenciada. Tampoco se tuvieron en cuenta aquellas publicaciones sin un resumen en inglés, ya que no podían ser analizadas por los investigadores, sin embargo, en la actualidad éstas representan una minoría. Como fortaleza, se encontraron pocas publicaciones similares a la actual. Una de las más importantes es la revisión de Cochrane de 2012 (16), en la cual se evaluaba el efecto de la adherencia al CONSORT en la completitud del reporte de los ensayos clínicos, cuya conclusión fue que en aquellas revistas que adoptaban el CONSORT sí mejoraba la calidad del reporte. En la revisión de Susvirkar et al de 2018 (18), evaluaron la adherencia de los artículos sobre ensayos clínicos publicados en dos revistas de alto impacto, el Journal of the American Medical Association y el British Medical Journal a las recomendaciones del CONSORT, la cual aunque cercana a $80 \%$ mostró pobre adherencia, entre otros, a los ítems de identificación como ensayo clínico en el título y descripción del diseño del mismo.

No se hallaron artículos en los cuales se evaluara el comportamiento de la publicación de ensayos clínicos por tipo de diseño, por lo cual este puede ser un punto de partida para nuevos estudios al respecto, y además para incentivar la adherencia a las recomendaciones del CONSORT, mejorando la calidad de la producción científica.

\section{Referencias}

1. Friedman LM, Furberg CD, Demets DL, Reboussin DM, Granger CB. Introduction to Clinical Trials. En: Fundamentals of Clinical Trials. 5th ed. Springer; 2015. p. 2.

2. Lilienfeld A. CETERIS PARIBUS. The evolution of the clinical trial. Bull Hist Med. 1982;56:1-18.

3. Argimon Pallás JM, Jiménez Villa J. Estudios experimentales I: el ensayo clínico aleatorio. En: Métodos de investigación clínica y epidemiológica. 4a ed. Barcelona: Elsevier España; 2013. p. 33-48.
4. Friedman LM, Furberg CD, Demets DL. Basic study design. En: Fundamentals of Clinical Trials. 5th ed. Springer; 2015. p. 89-121.

5. Argimon Pallás JM, Jiménez Villa J. Estudios experimentales II: otros diseños En: Métodos de investigación clínica y epidemiológica. 4a ed. Barcelona: Elsevier España; 2013.p. 49-63.

6. Guyatt G, Rennie D, Meade M, Cook D. Advanced topics in the validity of therapy trials: N-of-1 randomized controlled trials. En: Association AM, editor. Users' Guides to the Medical Literature, A Manual for Evidence-Based Clinical Practice. 2nd ed. Mc-Graw Hill; 2008. p. 179-92.

7. Pandis N, Chung B, Scherer RW, Elbourne D, Altman DG. CONSORT 2010 statement: extension checklist for reporting within person randomised trials. BMJ. 2017;180(3):j2835.

8. Mistry P, Dunn JA, Marshall A. A literature review of applied adaptive design methodology within the field of oncology in randomised controlled trials and a proposed extension to the CONSORT guidelines. BMC Med Res Methodol. 2017;17(1):1-9.

9. Zwarentein M, Treweek S, Gagnier JJ, Altman DG, Tunis S, Haynes B, et al. Improving the reporting of pragmatic trials: An extension of the CONSORT statement. J Chinese Integr Med. 2009;7(4):392-7.

10.Schulz KF, Altman DG, Moher D. CONSORT 2010 Statement: updated guidelines for reporting parallel group randomised trials. BMJ. 23 de marzo de 2010;340(mar23 1):c332-c332.

11. Marion KC, Diana RE, Douglas GA. CONSORT statement: extension to cluster randomised trials. BMJ. 2004;328.

12. Campbell MK, Piaggio G, Elbourne DR,Altman DG. Consort 2010 statement: Extension to cluster randomised trials. BMJ. 2012;345(7881):1-21

13. Piaggio G, Elbourne DR, Altman DG, Pocock SJ, Evans SJW. CONSORT Group for the. Reporting of Noninferiority and Equivalence Randomized Trials. JAMA. 8 de marzo de 2006;295(10): 1152 .

14. Piaggio G, Elbourne DR, Altman DG, Pocock SJ, Evans SJW. Reporting of noninferiority and equivalence randomized trials (for the CONSORT Group). $J$ Am Med Assoc. 2012;308(24):2594-604.

15. Vohra S, Shamseer L, Sampson M, Bukutu C, Schmid CH, Tate R, et al. CONSORT extension for reporting N-of-1 trials (CENT) 2015 Statement. BMJ. 14 de mayo de 2015;350(may14 17):h1738-h1738.

16. Turner, L., Shamseer, L., Altman, D. G., Schulz, K. F., \& Moher, D. Does use of the CONSORT Statement impact the completeness of reporting of randomised controlled trials published in medical journals? A Cochrane reviews. Systematic Reviews, 1(1), 60. https://doi.org/10.1186/2046-4053-1-60

17. Jin, Y., Sanger, N., Shams, I., Luo, C., Shahid, H.,Samaan, Z. Does the medical literature remain inadequately described despite having reporting guidelines for 21 years?-A systematic review of reviews: an update. Journal of Multidisciplinary Healthcare, Volume 11, 495-510. https://doi.org/10.2147/JMDH.S155103

18. Susvirkar, A., Gada, P., Figer, B., Thaker, S., Thatte, U., \& Gogtay, N. An assessment of the compliance of randomized controlled trials published in two high impact journals with the CONSORT statement. The National Medical Journal of India, 31(2), 79. https://doi.org/10.4103/0970-258X.253165

19. Lazcano-Ponce E, Salazar-Martínez E, Gutiérrez-Castrellón P, et al. Ensayos clínicos aleatorizados: variantes, métodos de aleatorización, análisis, consideraciones éticas y regulación. salud publica mex. 2004;46(6):559-584.

20. Reveiz Ludovic, Villanueva Eleana, Iko Chimaraoke, Simera Iveta. Compliance with clinical trial registration and reporting guidelines by Latin American and Caribbean journals. Cad. Saúde Pública [Internet]. 2013 June [cited 2020 Nov 03] ; 29( 6 ): 1095-1100. Available from: http://www.scielo.br/scielo. php?script=sci_arttext\&pid=S0102-311X2013000600006\&lng=en. https://doi. org/10.1590/S0102-311X2013000600006.

21. Cohen J.A coefficient of agreement for nominal scales. Educ Psychol Meas 1960; 20: 37-46. https://doi.org/10.1177/2F001316446002000104

22. Manterola Carlos, Grande Luis, Otzen Tamara, García Nayely, Salazar Paulina, Quiroz Guissela. Confiabilidad, precisión o reproducibilidad de las mediciones. Métodos de valoración, utilidad y aplicaciones en la práctica clínica. Rev. chil. infectol. [Internet]. 2018 [citado 2020 Nov 03] ; 35( 6 ): 680-688. Disponible en: https://scielo.conicyt.cl/scielo.php?script=sci_ arttext\&pid=S0716-10182018000600680\&lng=es. http://dx.doi.org/10.4067/ S0716-10182018000600680.

22. Epidat: programa para análisis epidemiológico de datos. Versión 4.2, julio 2016 Consellería de Sanidade, Xunta de Galicia, España; Organización Panamericana de la salud (OPS-OMS); Universidad CES, Colombia. 


\section{Material suplementario}

- Ensayo clínico aleatorizado: randomized controlled trial, filtro: Randomized Controlled Trial, Fecha de publicación de 1979/01/01 a 2018/12/31.

- Grupos paralelos: parallel group[Title/Abstract], filtro: Randomized Controlled Trial, Fecha de publicación de 1979/01/01 a 2018/12/31.

- Asignación por grupos: cluster randomised[Title/Abstract] filtro: Randomized Controlled Trial, Fecha de publicación de 1979/01/01 a 2018/12/31.

- Cruzado: filtro: Randomized Controlled Trial, Fecha de publicación de 1979/01/01 a 2018/12/31.

- $\quad \mathbf{N}$ de 1: $n$ of 1[Title/Abstract], filtro: Randomized Controlled Trial, Fecha de publicación de 1979/01/01 a 2018/12/31.

- Factorial: factorial trial[Title/Abstract] OR factorial study[Title/Abstract] OR factorial design[Title/ Abstract] OR factorial randomised[Title/Abstract], filtro: Randomized Controlled Trial, Fecha de publicación de 1979/01/01 a 2018/12/31.

- Pareado: "Within person design"[Title/Abstract] OR "Within person trial"[Title/Abstract] OR "Splitmouth design"[Title/Abstract] OR "Split-mouth trial"[Title/Abstract] OR "Split-face design"[Title/ Abstract] OR "Split-face trial"[Title/Abstract] OR "Split-body design"[Title/Abstract] OR "Splitbody trial"[Title/Abstract] OR "Contralateral design"[Title/Abstract] OR "Contralateral trial"[Title/ Abstract] OR "Matched pair design"[Title/Abstract] OR "Matched pair trial"[Title/Abstract] OR "Pair-matching design"[Title/Abstract] OR "Pair-matching trial"[Title/Abstract] OR "Randomized paired design"[Title/Abstract] OR "Randomized paired trial"[Title/Abstract] OR "randomised paired design"[Title/Abstract] OR "randomised paired trial"[Title/Abstract] OR "Within person study"[Title/Abstract] OR "Within person studies"[Title/Abstract] OR "Split-mouth study"[Title/ Abstract] OR "Split-mouth studies"[Title/Abstract] OR "Split-face study"[Title/Abstract] OR "Splitface studies"[Title/Abstract] OR "Split-body study"[Title/Abstract] OR "Split-body studies"[Title/ Abstract] OR "Contralateral study"[Title/Abstract] OR "Contralateral studies"[Title/Abstract] OR "Matched pair study"[Title/Abstract] OR "Matched pair studies"[Title/Abstract] OR "Pairmatching study"[Title/Abstract] OR "Pair-matching studies"[Title/Abstract] OR "Randomized paired study"[Title/Abstract] OR "Randomized paired study"[Title/Abstract] OR "randomised paired studies"[Title/Abstract] OR "randomised paired studies"[Title/Abstract]; filtro: Randomized Controlled Trial, Fecha de publicación de 1979/01/01 a 2018/12/31.

- De retiro: withdrawal study[Title/Abstract] OR withdrawal trial[Title/Abstract] OR withdrawal design[Title/Abstract] filtro: Randomized Controlled Trial, Fecha de publicación de 1979/01/01 a $2018 / 12 / 31$.

- Adaptativo: adaptive randomised[Title/Abstract] OR adaptive trial[Title/Abstract] OR adaptive study[Title/Abstract] OR adaptive design[Title/Abstract]; filtro: Randomized Controlled Trial, Fecha de publicación de 1979/01/01 a 2018/12/31.

- $\quad$ Pragmático: pragmatic trial[Title/Abstract] OR pragmatic randomised[Title/Abstract] OR pragmatic study[Title/Abstract] OR pragmatic design[Title/Abstract]; filtro: Randomized Controlled Trial, Fecha de publicación de 1979/01/01 a 2018/12/31.

- Superioridad: superiority[Title/Abstract]; filtro: Randomized Controlled Trial, Fecha de publicación de 1979/01/01 a 2018/12/31.

- No inferioridad: noninferiority[Title/Abstract]; filtro: Randomized Controlled Trial, Fecha de publicación de 1979/01/01 a 2018/12/31.

- Equivalencia: equivalence[Title/Abstract]; filtro: Randomized Controlled Trial, Fecha de publicación de 1979/01/01 a 2018/12/31. 